\title{
Survey of X-ray pulsars in hard X-rays
}

\author{
Alexander Lutovinov* \\ Space Research Institute, Russia \\ E-mail: aaldiki.rssi.ru
}

\section{Sergey Tsygankov}

MPI for Astrophysics, Munich, Germany;

Space Research Institute, Moscow, Russia

E-mail: tsygankov@iki.rssi.ru

\begin{abstract}
Results of spectral, timing and spatial analysis of X-ray pulsars in hard X-rays are briefly discussed. Using data of INTEGRAL, XMM-Newton and Chandra observatories we determined an optical counterparts and distances for several X-ray pulsars and built the spatial distribution of this class objects in the Galaxy. Variations of the cyclotron line energy with the luminosity, orbital and pulse phases were studied in a complex manner. In particular, an evolution of $\mathrm{V} 0332+53$ spectral parameters during the brightening and fading phases of the outburst in 2004-2005 shows no evidence for any hysteretic behavior and to impose the more stringent constraints on the magnetic field in the sources. Preliminary results of the pulse phase-resolved spectroscopy of the V $0332+53$ emission are presented.
\end{abstract}

The Extreme sky: Sampling the Universe above $10 \mathrm{keV}$

October 13-17 2009

Otranto (Lecce) Italy

\footnotetext{
${ }^{*}$ Speaker.
} 


\section{Introduction}

During about of 7 years of the operation in the orbit the INTEGRAL observatory [1] has detected more that $50 \mathrm{X}$-ray pulsars (including several new ones) at a high significance level, that gave us a possibility to study their properties in hard X-rays in details. Corresponding reviews can be found in papers of [⿰月/ (spectra) and [3] (timing analysis of ten brightest pulsars). For several sources it was possible to detect and study the cyclotron absorption features and carry out the detailed analysis of the spectral variability depending on the source luminosity and pulse phase.

Below we summarize briefly current results of observations of X-ray pulsars, drawing the main attention to their spatial distribution, the search for the hysteresis behavior of spectral parameters during outbursts and pulse-resolved spectroscopy.

\section{Spatial distribution of X-ray pulsars}

We are continuing a study of the Galaxy structure in hard X-rays ( $>20 \mathrm{keV})$ using data of the INTEGRAL observatory (see, e.g., [4, 5. 6] and references therein). Observations and discoveries of a large number X-ray sources allowed us to improve significantly our knowledge about different populations of X-ray binaries, their properties and spatial distribution.

At the moment (October of 2009) a number of confidently detected with the INTEGRAL observatory sources is 488 ([7), 84 of which are high-mass X-ray binaries (HMXBs) and 93 are low-mass X-ray binaries (LMXBs). It is known that HMXBs and LMXBs distributions in the Galaxy have different vertical scale heights, roughly correspond to 2 and 5 degrees from the galactic plane at a distance of the Galactic Center (GC), respectively [8]. Following to [6] we present below two current samples of HMXBs and X-ray pulsars with $b<|2|$ deg and $b<|5|$ deg from the Galactic plane (see Table below and Fig.11).

$\begin{array}{llll}\text { HMXB: } & 84 \text { sources } & \text { X-ray pulsars: } & 52 \text { sources } \\ |b|<5^{\circ} & 78 \text { sources } & |b|<5^{\circ} & 46 \text { sources } \\ |b|<2^{\circ} & 63 \text { sources } & |b|<2^{\circ} & 39 \text { sources }\end{array}$

The majority of X-ray pulsars belong to the class of HMXBs therefore it is not a surprise that both distributions are very similar - as well as HMXBs, X-ray pulsars are concentrated towards the Galactic plane and mostly located in spiral arms as a young galactic population. But it is interesting to note two points: 1) an increase of a number of HMXBs (and consequently, X-ray pulsars) in the GC direction (see, e.g., Fig.1] and Fig.6 in [円) and 2) some skewness of the X-ray pulsars distribution along the galactic plane in a comparison with the HMXBs one: 20 pulsars to the "left" of GC vs 26 pulsars to the "right" of GC and 39 vs 39 for HMXBs, respectively. The former is connected with the increasing sensitivity of our X-ray observations and optical identifications of sources located behind GC, possibly in further parts of spiral arms (see, e.g., [9] for details). Reasons for the second are not fully clear and require additional investigations. 


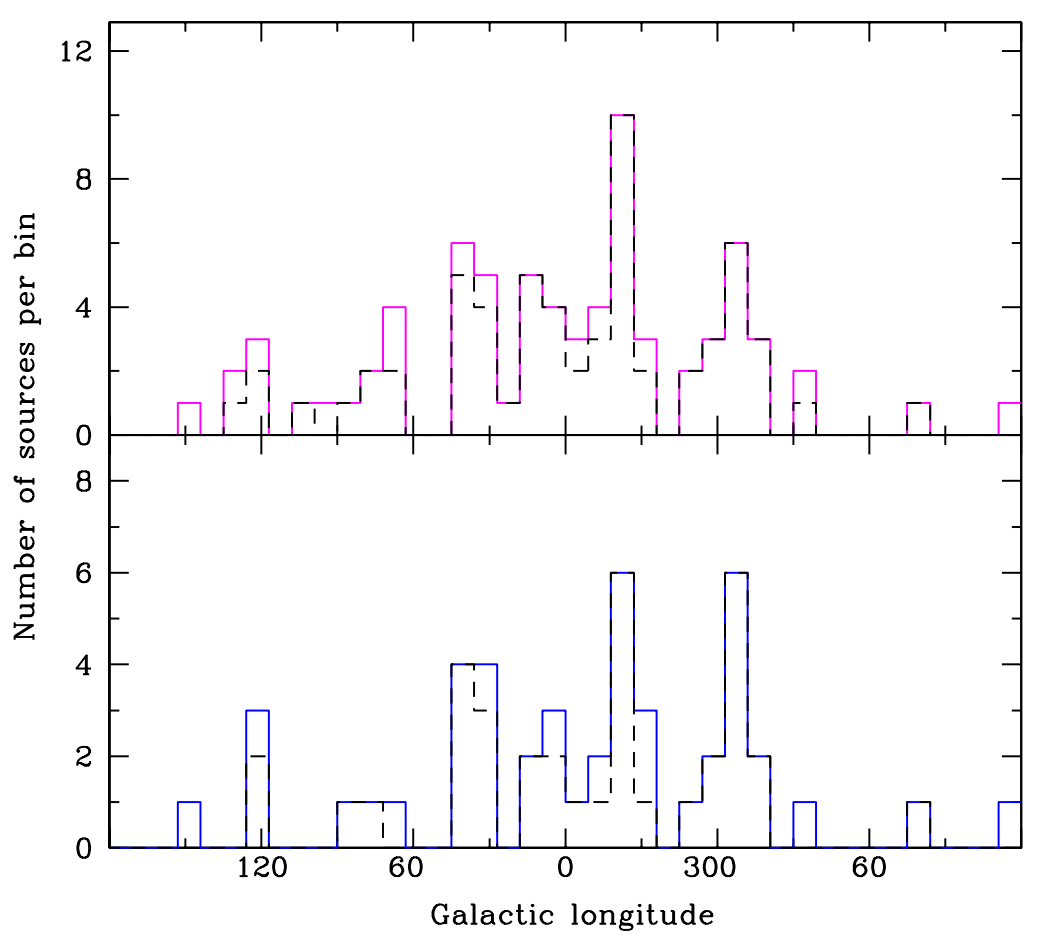

Figure 1: Spatial distribution of HMXBs (upper panel) and X-ray pulsars (bottom panel) along the galactic plane. Solid lines represent the number of sources with the latitude of $|b|<5^{\circ}$, dashed lines - sources with the latitude of $|b|<2^{\circ}$.

\section{Bright X-ray pulsars in outbursts: search for the hysteretic behavior}

It was known before that the energy of the cyclotron absorption line can somehow depends on the source luminosity (see, e.g., [10]). Using INTEGRAL and RXTE data, obtained during the fading phase of the 2004-2005 outburst, Tsygankov et al. [11] showed for the first time that the energy of the cyclotron line fundamental harmonic in the $\mathrm{V} 0332+53$ spectrum grows approximately linearly with the decreasing the source luminosity. Including both the fading and brightening phases of the outburst into an analysis made it possible to study the possible difference in the cyclotron-line behavior during these outburst stages and unambiguously ruled out the hysteresis hypothesis [12]. Fig. Da a clearly demonstrates that the dependence of the cyclotron line energy on the luminosity is exactly the same for both brightening and fading phases of the outburst (at least at luminosities higher than $\sim 1.5 \times 10^{38} \mathrm{erg} / \mathrm{s}$ ). Hence, the physics of the accretion column and its emission is essentially same at both these stages. In approaching the dipole magnetic field of the neutron star, the maximum measured change of the cyclotron line energy corresponds to $\sim 1 \mathrm{~km}$ of the relative change of the height of its formation above the neutron star surface. The lowest observed value of the cyclotron energy corresponds to the magnetic field on the neutron-star surface $B \simeq 3.5 \times 10^{12} \mathrm{G}$, that is slightly higher than considered earlier (the neutron star radius and mass were taken as $10 \mathrm{~km}$ and $1.4 M_{\odot}$, respectively).

Another well known transient X-ray pulsar 4U 0115+63 was studied with INTEGRAL and 

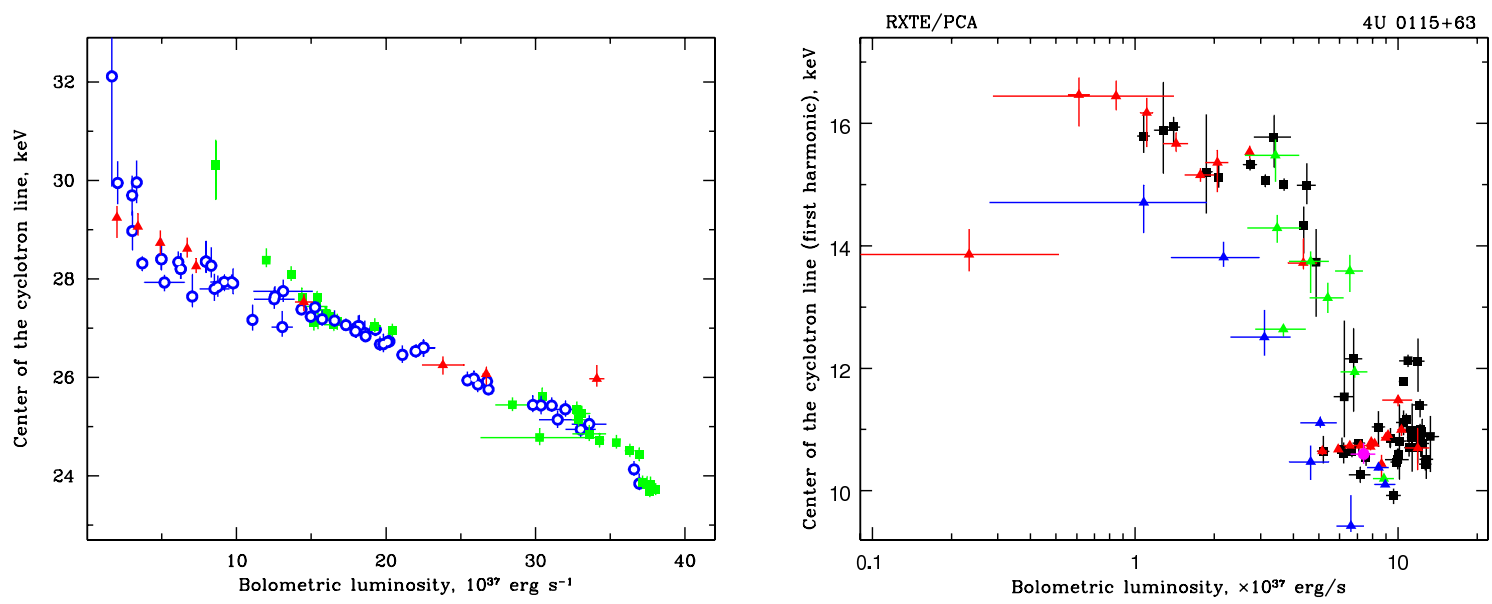

Figure 2: (a) V 0332+53 cyclotron line energy dependence on the luminosity. Filled green squares and open blue circles correspond to RXTE measurements in the brightening and fading phases, respectively; red triangles are INTEGRAL results. (b) The same, but for 4U 0115+63: black squares and red triangles are RXTE measurements performed during outbursts of 1999 and 2004, respectively; a magenta circle is the INTEGRAL measurement in 2004. RXTE measurements in the brightening and fading phases of the 2008 outburst are shown by green and blue tringles, respectively.

RXTE observatories during its intense outbursts in 1999 and 2004 [13, 14]. As contrasted with $\mathrm{V} 0332+53$, the energy of the cyclotron line fundamental harmonic of $4 \mathrm{U} 0115+63$ is practically constant $(\sim 11 \mathrm{keV})$ in the high luminosity state $\left(5 \times 10^{37}-2 \times 10^{38} \mathrm{erg} \mathrm{s}^{-1}\right)$; but when the pulsar luminosity falls below $\sim 5 \times 10^{37} \mathrm{erg} \mathrm{s}^{-1}$, the fundamental harmonic energy is displaced sharply towards the high energies, up to $\sim 16 \mathrm{keV}$ (Fig.2 $2 \mathrm{~b}$ ). For the dipole magnetic field configuration, this change in the cyclotron energy corresponds to the decrease of the height of its formation region by $\sim 2 \mathrm{~km}$. Note the similarity of a cyclotron line behavior in 1999 and 2004 outbursts.

A preliminary analysis of a new outburst from $4 \mathrm{U} 0115+63$ in 2008 allowed us to trace cyclotron line changes during both brightening and fading phases (Fig. 2 $b$ ). Some indications to a possible hysteresis are seen from a figure, in line with a general similarity of the line energy dependence on the source luminosity with previous outbursts.

\section{Pulse phase-resolved spectroscopy}

It is well known that some spectral features of the X-ray pulsars emission could be explored using only a pulse phase-resolved spectral analysis. It deals with a difference in physical conditions of different parts of emission regions and dependence of the emission observable properties on the viewing angle (e.g. [15] and references therein). As it was shown for V $0332+53$, its pulse profile is highly variable with the energy and luminosity [11, 12] and should reflect the source spectrum dependence on the pulse phase.

First attempts to perform a phase-resolved spectroscopy for V 0332+53 were undertake by Pottschmidt et al. [16] using the RXTE data from several bright observations at the outburst declining phase. But, at that moment only the PCA/RXTE data without a sufficient energy and temporal resolution were available in the $3-20 \mathrm{keV}$ energy band. Therefore such an analysis was 

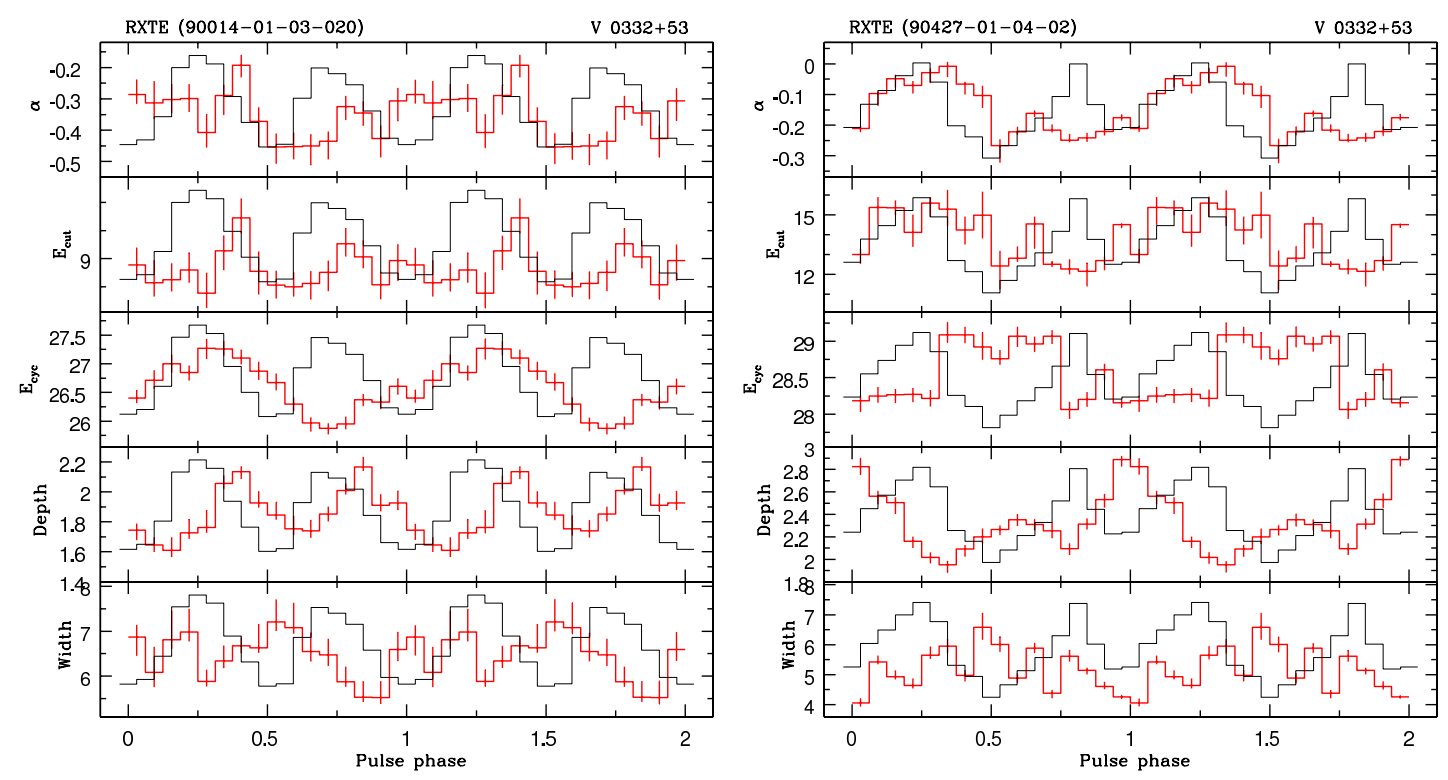

Figure 3: Variability of continuum and cyclotron line parameters (red histograms) with the V $0332+53$ pulse phase for two different values of the source luminosity. Corresponding mean pulse profiles in the 20-100 energy band are showen by black line.

done only in hard X-rays $(>20 \mathrm{keV})$ and showed insignificant variations of spectral parameters with the pulse phase. An inclusion into a consideration of a whole data set of V 0332+53 observations allowed us to carry out the pulse phase-resolved spectroscopy of the V $0332+53$ emission in a wide range of luminosities and energies (see for details Lutovinov, Tsygankov, prepared for the publication). In Fig. $3(a, b)$ variations of the continuum spectral parameters (photon index and cutoff energy) and parameters of the cyclotron line are shown in line with corresponding source pulse profiles for two different values of the luminosity, $\sim 1.6 \times 10^{38}$ and $\sim 6 \times 10^{37} \mathrm{erg} / \mathrm{s}$, respectively (for the approximation of phase-resolved spectra we used the same model as for the analysis of pulse-average spectra [12]). The major result of this analysis is the registration of changes of cyclotron line parameters (mainly, the line energy and its depth) during the pulse. One of possible explanations is that during different phases of the X-ray pulse regions with different magnetic field and under different viewing angle are observed. On the other side the spectral parameter changes during the pulse can be also connected with the variability of the system geometry and properties of the matter around the X-ray source (see [17] for details). Note, that variantions of a cyclotron line energy were found before for several other X-ray pulsars: 4U 0115+63 [18], Cen X-3 [19], Vela X-1 [20], etc., but no a generally plausible explanation was proposed to date.

\section{ANKNOWLEGEMENTS}

Authors thank to R.Krivonos for the help with a construction of spatial distributions. This work was partially supported by the Program "The origin, structure, and evolution of objects of the Universe" of the Russian Academy of Sciences and grant NSh-5069.2010.2 for support of leading scientific schools. 


\section{References}

[1] Winkler C., Courvoisier T., Di Cocco G., et al., Astronomy and Astrophysics, 411, (L1), 2003

[2] Filippova E., Tsygankov S., Lutovinov A., Sunyaev R., Astron. Letters, 31, (729), 2005

[3] Lutovinov A., Tsygankov S. Astron. Letters, 35, (433), 2009

[4] Lutovinov A., Revnivtsev M., Gilfanov R., et al., Astronomy and Astrophysics, 444, (821), 2005

[5] Krivonos, R., Revnivtsev, M., Lutovinov, A., et al., Astronomy and Astrophysics, 475, (775), 2007

[6] Lutovinov, A., Revnivtsev, M., Gilfanov, M., Sunyaev, R., Proceedings of the 6th INTEGRAL Workshop (Edited by S.Grebenev, R.Sunyaev, C.Winkler), ESA SP-622, (241), 2007, [arXiv:0801.3589]

[7] Krivonos, R., et al., Astronomy and Astrophysics, submitted

[8] Grimm, H.-J., Gilfanov, M. \& Sunyaev, R., Astronomy and Astrophysics, 391, (923), 2002

[9] Karasev D., Lutovinov A., Tsygankov S., Burenin R., Proceedings of the Workshop "The Extreme sky: Sampling the Universe above $10 \mathrm{keV"} \mathrm{(Eds.} \mathrm{P.Ubertini)} \mathrm{PoS,} \mathrm{96,} \mathrm{103,} \mathrm{2010,} \mathrm{this} \mathrm{issue}$

[10] Mihara T., Makishima K., Nagase F., Adv. Space Res., 22, (987), 1998

[11] Tsygankov S., Lutovinov A., Churazov E., Sunyaev R., MNRAS, 371, (19), 2006

[12] Tsygankov S., Lutovinov A., Serber A., MNRAS, 401, (1628), 2010

[13] Nakajima M., Mihara T., Makishima K., Niko H., The Astrophysical Journal, 646, (1125), 2006

[14] Tsygankov S., Lutovinov A., Churazov E., Sunyaev R., Astron. Letters, 33, (368), 2007

[15] Suchy S., Pottschmidt K., Wilms J., et al., The Astrophysical Journal, 675, (1487), 2008

[16] Pottschmidt K., Kreykenbohm I., Wilms J., et al., ApJ, 634, (L97), 2005

[17] Tsygankov S., Lutovinov A., Proceedings of the Workshop "The Extreme sky: Sampling the Universe above $10 \mathrm{keV"} \mathrm{(Eds.} \mathrm{P.Ubertini)} \mathrm{this} \mathrm{issue,} \mathrm{2010,} \mathrm{[arXiv:1002.1898]}$

[18] Santangelo A., Segreto A., Giarrusso S., et al., ApJ, 523, (L85), 1999

[19] Burderi L., Di Salvo T., Robba N., et al., ApJ, 530, (429), 2000

[20] Kreykenbohm I., Coburn W., Wilms J., et al., Astronomy and Astrophysics, 395, (129), 2002 